\title{
Acute Unstable Posterior Shoulder Dislocation Treated by Modified McLaughlin Procedure: A Case Report
}

\section{W Bouaicha*, M Jlidi, S Daas, K Charfi, MA Sbai and A Khorbi}

Orthopedics Department, Hospital MTM Nabeul, Tunisia

*Corresponding Author: W Bouaicha, Orthopedics Department, Hospital MTM Nabeul, Tunisia.
Received: January 25, 2021

Published: February 19, 2021

(C) All rights are reserved by $\mathbf{W}$ Bouaicha., et al.

\section{Abstract}

Posterior shoulder fracture-dislocation is a rare injury accounting for only $0.9 \%$ of all shoulder fracture-dislocations. The size of the associated “Reverse Hill-Sachs lesion (RHSL)” dictates the treatment options.

Several surgical techniques have been described for the treatment of this condition based on the chronicity of the injury and the size of the RHSL.

This article describes the use of a modified Mc Laughlin procedure in the treatment of an acute unstable posterior shoulder fracture dislocation in a 47-year old male. The surgical technique consists in transferring the subscapularis tendon after osteotomy of its lesser tubercle insertion into the anterior bony defect. This transfer is secured with screw fixation and transosseous suturing. Post operative rehabilitation included 4 weeks of immobilization followed by 6-weeks progressive physiotherapy.

This technique yielded a pain-free and stable shoulder with good joint congruency.

Keywords: Shoulder; Posterior Fracture-dislocation; Reverse Hill-Sachs Lesion; Mc Laughlin Procedure

\section{Introduction}

Posterior shoulder fracture-dislocation is a rare injury accounting for only $0.9 \%$ of all shoulder fracture-dislocations $[1,2]$.

Mechanisms of occurrence of this traumatic entity involve either a violent muscle contraction such as in epileptic seizures and electric shocks [3-7] or a major trauma in patients involved in motor vehicle accidents (MVA) or other injuries involving axial loading of the arm in an adducted, flexed and internally rotated position [3].

Impression fractures of the articular surface of the humeral head, the so-called "Reverse Hill Sachs Lesion", were the most commonly associated fractures (29\%) [7,8].
Often misdiagnosed ( $50 \%$ to $75 \%$ ), posterior fracture-dislocations of the shoulder are of a significant diagnostic and therapeutic interest because of its predilection for age groups of high functional demand $[9,10]$.

Treatment options vary and depend on the size of the humeral head defect, the duration of the dislocation and the degree of instability [11]. Several techniques have been described for the management of this injury, including disimpaction/bone grafting, sub-scapularis tendon transfer into the defect with or without the lesser tuberosity, rotation osteotomy of the proximal humerus and hemi or total shoulder arthroplasty [11-13]. 
This article reports the case of a patient with unstable acute posterior shoulder dislocation treated with a modified McLaughlin technique.

\section{Materials and Methods}

We report the case of a 47-year old male patient who presented to the emergency department with the chief complaint of a painful right shoulder following an injury on duty (fall from a $2 \mathrm{~m}$ high).

The diagnosis of a posterior shoulder dislocation was suspected based on the findings of plain x-rays of the shoulder showing the classic "light bulb sign" appearance associated with a defect in the anterior aspect of the humeral head on axial view (Figures 1,2).

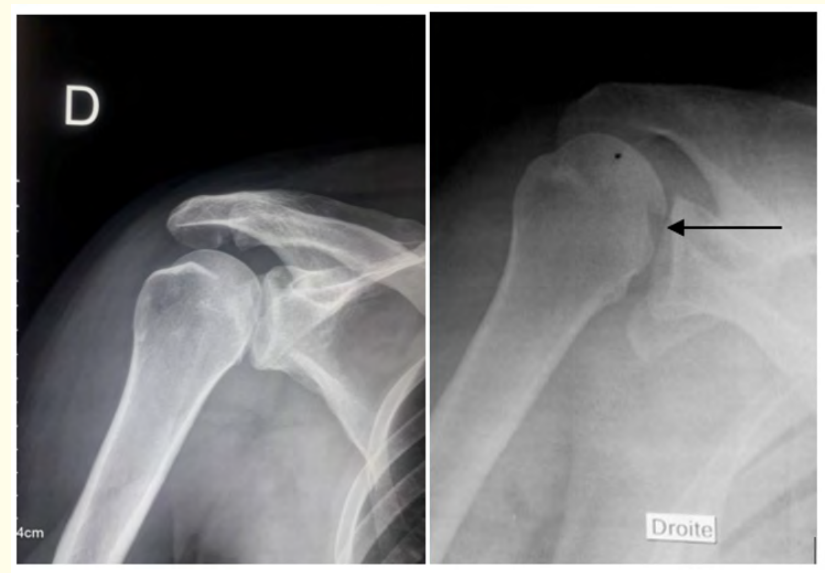

Figures 1,2: Initial X-rays showing the "light bulb sign"

(Figure1) and the reverse Hill-Sachs lesion (black arrow Figure 2).

Further investigation with a Computed Tomography (CT) scan imaging confirmed the diagnosis of a posterior fracture-dislocation of the shoulder with a reverse Hill-Sachs lesion involving about $25 \%$ of the humeral head (Figure 3 ).

Closed reduction under general anaesthesia failed after several attempts because of joint instability with the humeral head falling back posteriorly as soon as the reducing forces are released.

The patient was then put in the "beach chair" position with sterile draping of the right upper limb allowing for mobilisation of the shoulder in all directions.

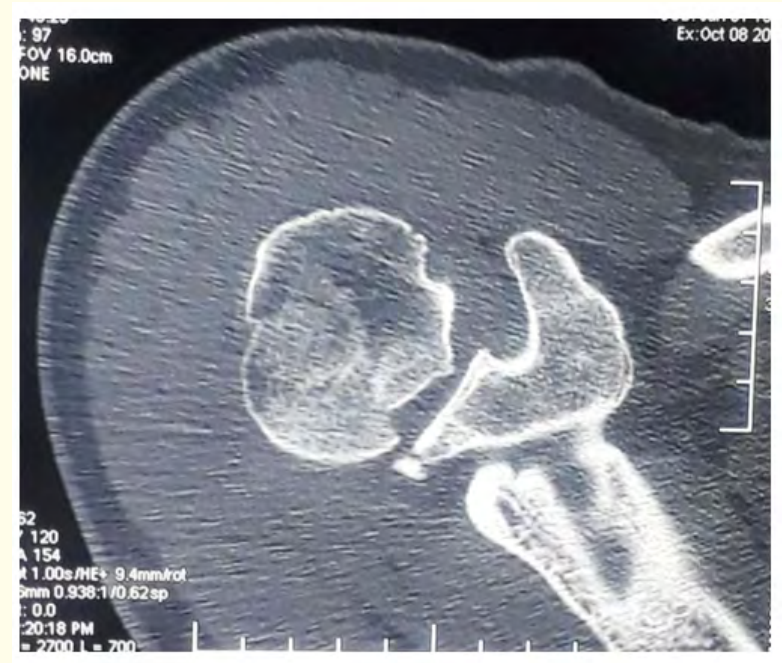

Figures 3: CT scan imaging.

A Neer modification of the original McLaughlin technique was used, consisting in an open reduction of the dislocated shoulder through an anterior deltopectoral approach. After reducing the shoulder, an osteotomy of the lesser tubercule is then made followed by a transfer of the subscapularis muscle together with its insertion on the lesser tuberosity into the bony defect of the humeral head. The transfer is then fixed with two $3.5 \mathrm{~mm}$ cancellous screws. A trans-osseous nonabsorbable \#2 suture was also made through the myotendinous junction of the sub scapularis muscle to further secure the transfer (Figures 4,5).

Stability of the shoulder joint was then assessed per-operatively with direct visualisation and fluoroscopy through the entire range of motion and transfixation of the glenohumeral joint with K-wires was not necessary.

Total operative time was 80 minutes. Preoperative antibiotic prophylaxis was given with double dose of Amoxicilline-Clavulanic acid.

Post-operatively, the shoulder was immobilised in abductionexternal rotation (ER) for 4 weeks using a shoulder sling with an ER pillow. Physiotherapy was then initiated with passive, active assisted and active range of motion. 


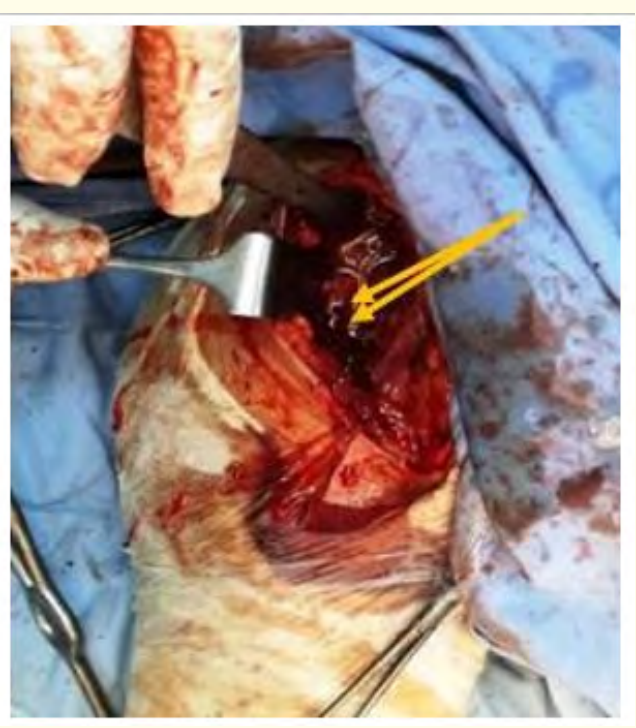

Figures 4: Open reduction and fixation of the lesser tubercle with two screws (Arrows).

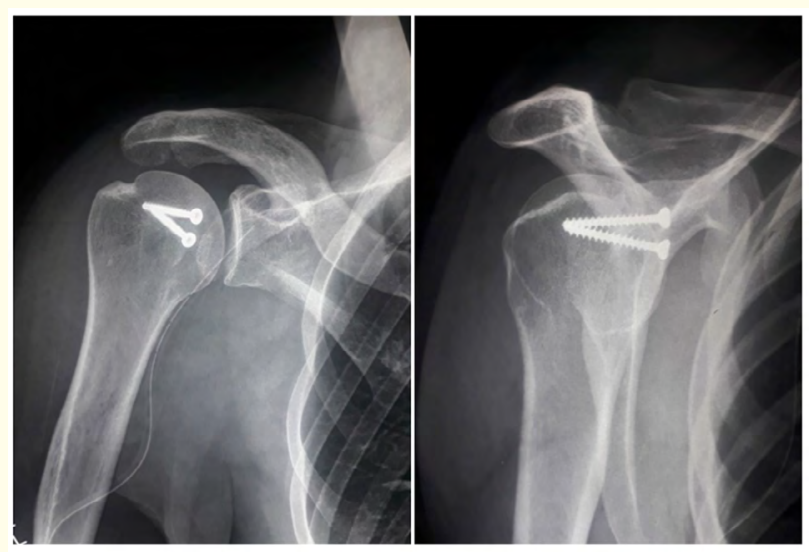

Figures 5: Post-operative X-ray (AP and Lateral).

At the last follow-up (8 months), plain x-ray shows a well reduced joint (Figure 6). The Constant and ASES scores were 80 and 71 respectively. The global shoulder range of motion was: $130^{\circ}$ forward flexion, $100^{\circ}$ abduction and $40^{\circ}$ external rotation. Patient visual satisfaction scale was $80 \%$ only reporting inconstant moderate pain.

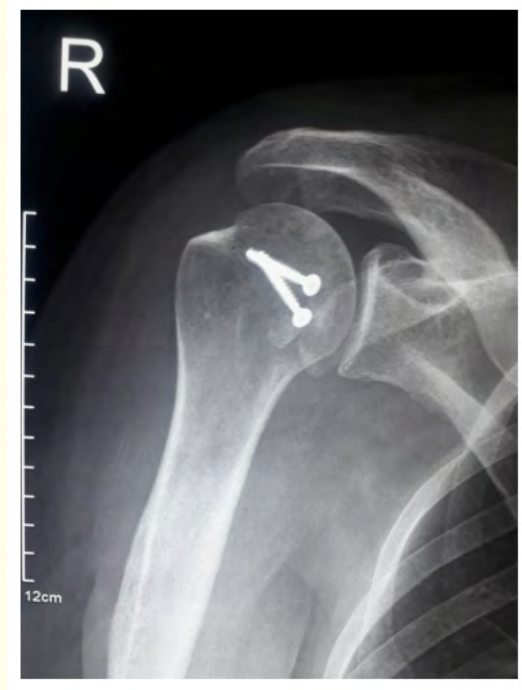

Figures 6: X-ray at the last follow-up (8 months).

\section{Discussion}

Posterior dislocation of the shoulder is a rare injury accounting for only 2-5 \% of all shoulder dislocations [1]. Even less frequent, posterior fracture-dislocation represents $0.9 \%$ of shoulder fracture-dislocations, occurring annually in $0.6 / 100.000$ people $[2,7]$.

Although rare, misdiagnosing this entity leads to severe functional impairment in the relatively young age groups in which it occurs (35 to 55years old), this lead McLaughlin to call it a "diagnostic trap" [5]. Moreover, a delayed diagnosis compromises vascularity and extends the head defect making final management trivial [14]. A careful history and clinical assessment, combined with a high index of suspicion should lead to accurate diagnosis [15]. Typical finding in physical examination would be limited active and passive external rotation [9] with the shoulder blocked in different degrees of internal rotation. A complete radiographic evaluation of the shoulder should include antero-posterior (AP) view, scapular lateral and axillary view as suggested by Neer [16].

Nowadays, CT imaging should be considered a routine examination for both suspected and confirmed cases [8,17]. It allows for determining the size of the humeral defect as well as detecting any additional fracture lines or fragments [1,7]. 
A thorough knowledge of the anatomy and biomechanics of the shoulder is important in the management of posterior shoulder fracture-dislocation. Regarding stability, impaction lesions of the articular surface of the humeral head determine the stable arc of curvature of the glenohumeral articulation which decreases when the size of the impression fracture increases [7]. A grading system was adopted to quantify the size of the reverse Hill-Sachs lesion: small $\leq 25 \%$, medium between 25 and $50 \%$ and large $\geq 50 \%$ [18]. Using this grading system our case fits in the medium size lesions with a depression involving at least a quarter of the humeral head. The size of the reverse Hill-Sachs lesion is thought to be the single most important factor for instability after posterior shoulder fracture-dislocation $[1,3]$ and is key to the decision process [18].

Mc Laughlin [5] was the first to recognize the importance of addressing the impaction fracture of the humeral head in posterior shoulder fracture-dislocations and his original procedure, described back in 1952, has been one of the most common surgical techniques to treat small to medium reverse Hill-Sachs lesions. This procedure consists in a tendon transfer of the subscapularis muscle into the bony defect.

Later, Hughes and Neer [19] as well as Hawkins., et al. [20] modified the original technique by osteotomizing the lesser tuberosity and transferring it with the attached subscapularis tendon into the bony defect. This modification was believed to provide better filling and a more secure fixation for the transfer. Several studies $[17,20,21]$ reported this technique with good results.

Other modifications of the original McLaughlin techniques have also been proposed with relatively good results: Charalambous., et al. [22] (plication of the subscapularis tendon into the defect), Spencer., et al. [23] (anchor suture of subscapularis tendon into defect without detachment from lesser tuberosity), Kokkalis., et al. [11] (bone allograft, lesser tuberosity osteotomy and anchor suture fixation on grafted defect).

The technique we used combined the screw fixation of the Hughes and Neer modification with the transosseous suture fixation of the Kokkalis technique.

However, these non-anatomical techniques have been criticised in the literature for altering the humeral head anatomy and limiting internal rotation of the shoulder and thus compromising a possible secondary prosthetic reconstruction [24-26].
To address this, Dubousset [26] was the first to suggest the use of autogenous iliac crest bone graft to restore the humeral head anatomy combined with posterior capsule reconstruction. Thirty years later, Gerber., et al. [25] published reports of 4 patients treated with allograft restoration of the humeral head without addressing the posterior capsulolabral tear.

In larger reverse Hill-Sachs lesions greater than $50 \%$ of the articular surface as well as in chronic dislocations older than 6 months, shoulder arthroplasty (SA), either hemi (HSA) or total (TSA), can be used as a last resort treatment option [7,20].

\section{Conclusion}

Posterior fracture-dislocations of the shoulder are rare yet important to diagnose to avoid severe joint functional disability.

Multiple treatment options are described in the literature.

This article describes a modified McLaughlin technique using screw fixation in adjunction with trans-osseous suture fixation in the treatment of unstable acute posterior fracture dislocation of the shoulder with a reverse Hill Sachs lesion of about $25 \%$. The final result was satisfactory with a mobile, pain free and stable shoulder joint.

\section{Bibliography}

1. Kowalsky MS and Levine WN. "Traumatic posterior glenohumeral dislocation: classification, pathoanatomy, diagnosis, and treatment". Orthopedic Clinics of North America 39.4 (2008): 519-533.

2. Robinson CM. "Complex posterior fracture-dislocation of the shoulder: epidemiology, injury patterns, and results of operative treatment". Journal of Bone and Joint Surgery 89.7 (2007): 1454.

3. Sheehan S and Gaviola G. "Traumatic shoulder injuries: a force mechanism analysis-glenohumeral dislocation and instability". American Journal of Roentgenology 201.2 (2013): 378-393.

4. Hawkins RJ and Belle RM. "Posterior instability of the shoulder". Instructional Course Lectures 38 (1989): 211-215.

5. McLaughlin HL. "Posterior dislocation of the shoulder". Journal of Bone and Joint Surgery 24.3 (1952): 584-590.

6. Kelly JP. "Fractures complicating electro-convulsive therapy and chronic epilepsy". Journal of Bone and Joint Surgery 36.1 
(1954): 70-79.

7. Kokkalis ZT., et al. "Posterior shoulder fracture-dislocation: an update with treatment algorithm". European Journal of Orthopaedic Surgery and Traumatology 27 (2017): 285-294.

8. Rouleau DM and Hebert-Davies J. "Incidence of associated injury in posterior shoulder dislocation: systematic review of the literature". Journal of Orthopadic Trauma 26.4 (2012): 246251.

9. Rowe CR and Zarins B. "Chronic unreduced dislocations of the shoulder". Journal of Bone and Joint Surgery 64.4 (1982): 494505.

10. Casado-Sanz E., et al. "Complex fractures of the proximal humerus". Complex fractures of the limbs. Springer, Cham (2014): 1-8.

11. Kokkalis ZT., et al. "Modified McLaughlin technique for neglected locked posterior dislocation of the shoulder". Orthopedics 36.7 (2013): e912-e916.

12. Cheng Sl., et al. "Treatment of locked posterior fracture-dislocations of the shoulder by total shoulder arthroplasty". Journal of Shoulder and Elbow Surgery 6.1 (1997): 11-17.

13. Porteous MJ and Miller AJ. "Humeral rotation osteotomy for chronic posterior dislocation of the shoulder". Journal of Bone and Joint Surgery 72.3 (1990): 468-469

14. Bock P., et al. "Anatomical reconstruction for reverse Hill-Sachs lesions after posterior locked shoulder dislocation fracture: a case series of six patients". Archives of Orthopaedic and Trauma Surgery 127.2 (2007): 543-548.

15. Sharma D., et al. "Asymmetrical fracture dislocation of shoulder-a case report and review of literature". Journal of Orthopaedic Case Reports 3.4 (2013): 19-22

16. Neer CS. "Displaced proximal humeral fractures. I. Classification and evaluation". Journal of Bone and Joint Surgery 52.6 (1970): 1077-1089

17. Castagna A., et al. "Modified MacLaughlin procedure in the treatment of neglected posterior dislocation of the shoulder". Chir Organi Mov 93 (2009): S1-5.

18. Cicak N. "Posterior dislocation of the shoulder". Journal of Bone and Joint Surgery 86.3 (2004): 324-332.
19. Hughes M and Neer CS. "Glenohumeral joint replacement and postoperative rehabilitation". Physical Therapy 55.8 (1975): 850-858

20. Hawkins RJ., et al. "Locked posterior dislocation of the shoulder". Journal of Bone and Joint Surgery 69.1 (1987): 9-18.

21. Robinson CM., et al. "Posterior shoulder dislocations and fracture-dislocations". Journal of Bone and Joint Surgery 87.3 (2005): 639-650.

22. Charalambous CP., et al. "A modification of the McLaughlin procedure for persistent shoulder instability: technical note". Archives of Orthopaedic and Trauma Surgery 129.6 (2009): 753-755

23. Spencer EE and Brems JJ. "A simple technique for management of locked posterior shoulder dislocations: report of two cases". Journal of Shoulder and Elbow Surgery 14.6 (2005): 650-652.

24. Checchia SL., et al. "Surgical treatment of acute and chronic posterior fracture dislocation of the shoulder". Journal of Shoulder and Elbow Surgery 7.1 (1998): 53-65.

25. Gerber C and Lambert SM. "Allograft reconstruction of segmental defects of the humeral head for the treatment of chronic locked posterior dislocation of the shoulder". Journal of Bone and Joint Surgery 78.3 (1996): 376-382.

26. Dubousset J. "Posterior dislocations of the shoulder [in French]". Revue de Chirurgie Orthopedique et Reparatrice de L'appareil Moteur 53.1 (1967): 65-85.

\section{Assets from publication with us}

- Prompt Acknowledgement after receiving the article

- Thorough Double blinded peer review

- Rapid Publication

- Issue of Publication Certificate

- High visibility of your Published work

Website: www.actascientific.com/

Submit Article: www.actascientific.com/submission.php Email us: editor@actascientific.com

Contact us: +919182824667 